\title{
CWS Compression: Cascading of Wavelet difference Reduction and Singular Value Decomposition
}

\author{
D.J. Ashpin Pabi \\ Research Scholar \\ Dept of Computer Science and Engineering \\ Annamalai University, Tamilnadu \\ India
}

\author{
P. Aruna, PhD \\ Professor \\ Dept of Computer Science and Engineering \\ Annamalai University, Tamilnadu \\ India
}

\begin{abstract}
People are sharing, transmitting and storing millions of images every day. To store images it may require huge data storage. The compression of images reduces the storage required to store images, also permits the faster transmission. Several works have been carried out in designing compression techniques that reduce image size with higher image quality. True color images take largest part in web pages, hence it is important to make control over image size and their quality to deliver fastest loading. This paper presents a new image compression technique CWS by cascading wavelet difference reduction (WDR) and singular value decomposition(SVD). In the proposed method, an input image is first compressed using WDR and then compressed using SVD. These two techniques are cascaded to boost the performance of WDR. The results are showing that the proposed compression is superior over the aforementioned compression techniques.
\end{abstract}

\section{Keywords}

Lossy Image Compression, Singular Value Decomposition, Wavelet Difference Reduction, Cascading.

\section{INTRODUCTION}

As digital images play an important role in transferring, sharing and storing visual information, the high compression techniques have been needed. One of the important advantages of compression is to speed up the transmission of images. Another one is to provide the compressed image at very high compression ratio without affecting the quality. The basic compression techniques can be categorized into lossy and lossless compressions. The quality of their output image shows the main differences. Lossy image compressions produce images with some degradation, which may be called visually lossless. The amount of data reduction using lossy compression is more substantial than lossless data compression. Such compression techniques are used to reduce the amount of data to store, handle, and or transmit the represented content. Even when the degradation is noticeable, the techniques are suitable for natural images such as photographs in application. The technique developed in this document is a lossy compression technique. The lossy image compression technique is developed using WDR followed by SVD

The paper has been arranged as: Section II describes the related works; section III describes the methodology of the proposed CWS compression technique. The next section shows its results and their discussions; finally the work has been concluded in section $\mathrm{V}$.

\section{RELATED WORKS}

As compression plays an important role in several areas, works have been carried out in developing efficient compression algorithms. Moonen, M. et al, (1992) developed a method which packs signal energy into fewer coefficients. Samruddhi Kahu et al, (2013) invented an image compression technique based on the Singular value decomposition (SVD). They mainly concentrated to reduce the number of eigen values required to reconstruct an image [1]. Konda, T.et al, (2009) described that the singular value decomposition is a part of a divide and conquer algorithm [2]. The method used by Andrews, H. C. et al, (1976) shows the application of SVD in digital images, also its importance in image processing and the point spread function representation [3]. Julie Kamm, L. (1998) developed a Newton method which is based on SVD to solve the problems occurs in signal and image processing applications [4]. Yang, J. F. et al, (1995) combined SVD and vector quantization (VQ) to achieve low bit rate and high quality image coding [5]. Anzhou $\mathrm{Hu}$ et al, (2014) predicted a single perceptual quality score by selecting local image distortion features using SVD [6]. Pratishtha Gupta et al, (2014) explained vector quantization finds the closest codeword for each image block in the codebook or dictionary [7]. Chien, C. S. et al, (2012) described an adaptive parametized block based singular value decomposition algorithm [8].

In this paper the wavelet difference reduction and singular value decomposition are cascaded to yield highly compressed images with good quality. First wavelet difference reduction is performed to the input image. The reconstructed image is applied to perform SVD. The comparisons over some existing algorithms are made by analyzing their performance measures.

\section{THE PROPOSED CWS IMAGE COMPRESSION TECHNIQUE}

\subsection{Wavelet Difference Reduction}

The WDR encoding combines run length encoding to produce an embedded image coder. The process of WDR compression technique is shown in Figure 1. The WDR algorithm is a simple procedure which comprises a bit-plane encoding technique. 


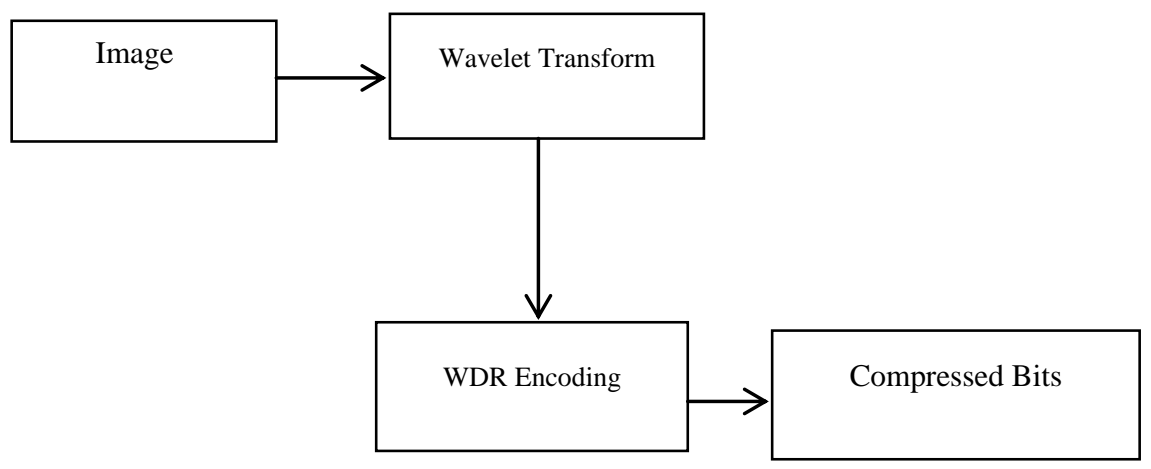

Figure 1: Flow diagram of the WDR compression technique

The steps of WDR algorithm includes:

1. A wavelet transform is applied to the input image.

2. The bit plane based WDR encoding algorithm has been carried out for the wavelet coefficients.

WDR mainly consists of the following four levels:

1. Initialization: An initial threshold $\mathrm{T}_{0}$ is chosen, such that, the transform $\left|\mathrm{x}_{\mathrm{m}}\right|<\mathrm{T}_{0}$ and at least one transform value satisfies $\left|\mathrm{x}_{\mathrm{m}}\right| \geq \mathrm{T}_{0} / 2$.

2. Threshold has to be updated, let $\mathrm{T}_{\mathrm{k}}=\mathrm{T}_{\mathrm{k}-1} / 2$

3. Significance Pass: Index values are encoded using the difference reduction method. The difference reduction method consists of a binary encoding of the number of steps between the index of the last significant value and the index of the current significant value. The signs of significant values along with the sequence of bits will be its output

4. Refinement Pass: Generates refined bits through the standard bit-plane quantization procedure.

\subsection{Singular Value Decomposition}

A color image takes three components, a red, a green and a blue (RGB). Hence, three times the space requires to store such images [9]. There are several ways to compress these images. One of which is singular value decomposition. This compress images by computing singular values. The mathematical expressions of singular value decomposition can be formulated as : Any nonzero real matrix A with rank $r>0$ can be decomposed into

$$
A=P \sum Q^{T}
$$

Where $\mathrm{P}$ is an $\mathrm{m} \times \mathrm{r}$ orthonormal column matrix

$$
\sum=\operatorname{diag}\left(\sigma_{1}, \sigma_{2}, \ldots \ldots, \sigma_{r}\right)
$$

$\mathrm{Q}^{\mathrm{T}}$ is an orthonormal row matrix
Such factorization is called the singular value decomposition. This is related to the spectral theorem, such that if $\mathrm{B}$ is a symmetric matrix $\left(\mathrm{B}^{\mathrm{T}}=\mathrm{B}\right)$, then

$\mathrm{B}=\mathrm{U} \wedge U^{T}$

Where $A$ is a diagonal matrix of eigenvalues

$\mathrm{U}$ is an orthonormal matrix of eigenvectors

To notice the relationship, consider:

$$
\begin{aligned}
& A^{T} A=Q \sum^{T} P^{T} P \sum Q^{T}=Q \sum^{2} Q^{T} \\
& A A^{T}=P \sum Q^{T} Q \sum^{T} P^{T}=P \sum^{2} P^{T}
\end{aligned}
$$

These two are separately decomposed and hence $\sigma_{\mathrm{i}}$ are the positive square roots of the eigen values. The matrices are rearranged, so that

$\sigma_{1} \geq \sigma_{2} \geq \cdots \geq \sigma_{n}$

Thus, an invertible matrix A with $\mathrm{n} \times \mathrm{n}$ can be written as:

$$
\begin{aligned}
& A=P \sum Q^{T}
\end{aligned}
$$

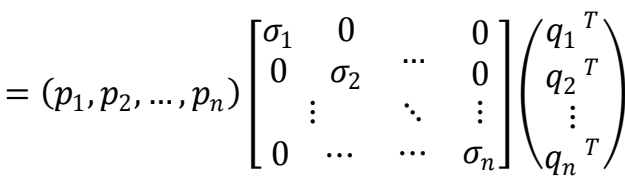

$$
\begin{aligned}
& =p_{1} \sigma_{1} q_{1}^{T}+p_{2} \sigma_{2} q_{2}^{T}+\cdots+q_{n} \sigma_{n} q_{n}^{T}
\end{aligned}
$$

Since $\sigma_{1} \geq \sigma_{2} \geq \ldots \ldots \ldots . . \sigma_{n}$, the terms $p_{\mathrm{i}} \sigma_{\mathrm{i}} \mathrm{q}_{\mathrm{i}}^{\mathrm{T}}$ with small $i$ contribute the sum, which contains the most information about the image. These terms result in a lower image quality, but lower storage size. As color images are in RGB, the reduced SVD can be computed on all three channels separately or together. 


\section{Encoding}

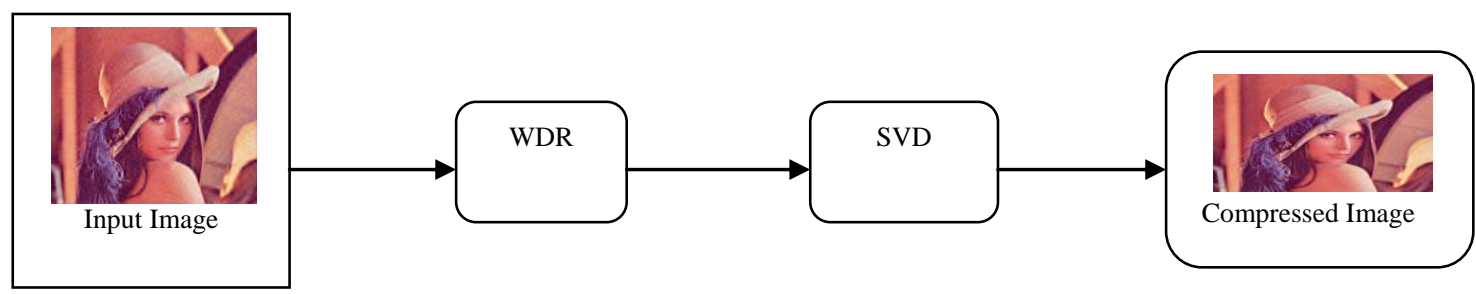

Decoding

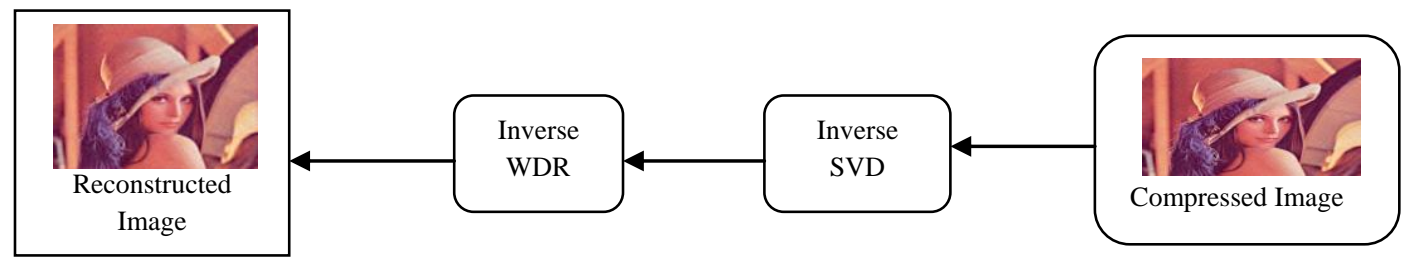

Figure 2: Block diagram of the proposed CWS compression technique

\subsection{The Proposed Methodology}

The block diagram of the proposed CWS image compression technique is shown in Figure 2. The proposed image compression technique is lossy, due to the nature of the SVD compression process. Already it is mentioned that SVD offers high PSNR values and low compression ratios.

The image is first compressed using WDR technique, which yields higher compression ratios. The loop for the WDR technique is fixed at 11. The reconstructed image is then decomposed using SVD, some singular values are ignored and then the image is reconstructed. At the end the overall compression ratio is calculated. The proposed CWS compression technique is lossy because of the nature of the process. The technique gets importance by cascading WDR compression with SVD compression.

\section{EXPERIMENTAL RESULTS AND DISCUSSIONS}

The proposed CWS lossy compression technique is applied to some true color images, including Lenna, Mandrill, Strawberry, City1, City2, Bus, Rose etc. The metrics such as peak signal-to-noise ratio (PSNR), the compression ratio (CR) and bits-per-pixel (bpp) [10] significantly predicts the performance of the proposed techniques with some already existing techniques. Figure 3 shows some of the test images used in this work.

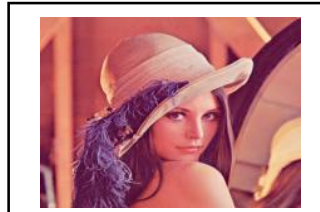

Lenna

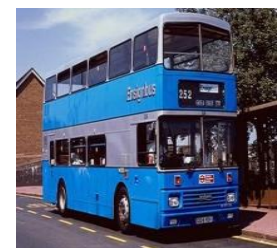

Bus

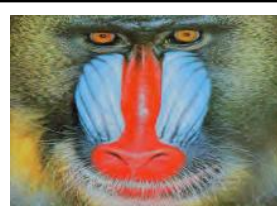

Mandrill

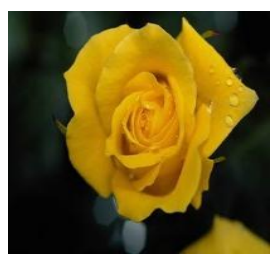

Rose

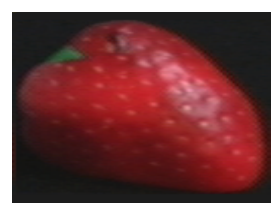

Strawberry

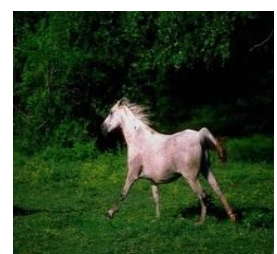

Horse

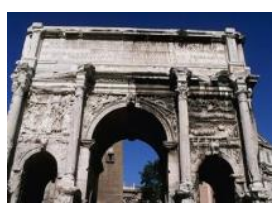

City1

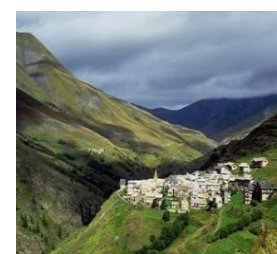

Mountain

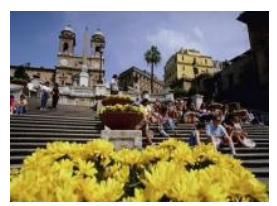

City2

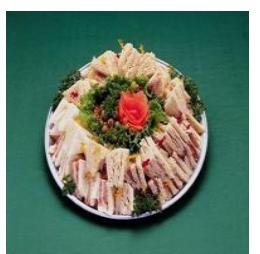

Food

Figure 3: Test Images 


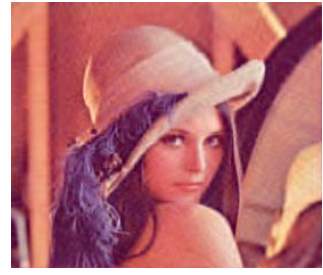

(a)

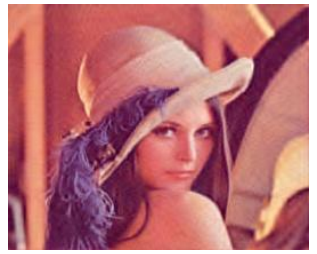

(c)

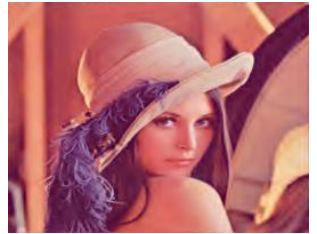

(e)

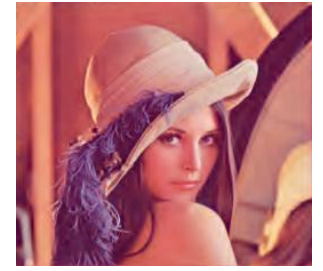

(b)

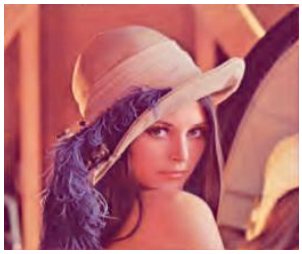

(d)

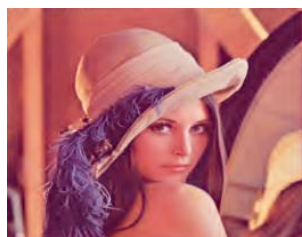

(f)
Figure 4: (a) Original image (b) Reconstructed images using the proposed CWS compression technique for sv: 40 (c) sv:50 (d) sv:100 (e) sv:200 (f) sv:300

Figure 4 shows the visual output of Lenna image, when the proposed technique is applied to various singular values such as 50,100, 200 and 300 respectively.

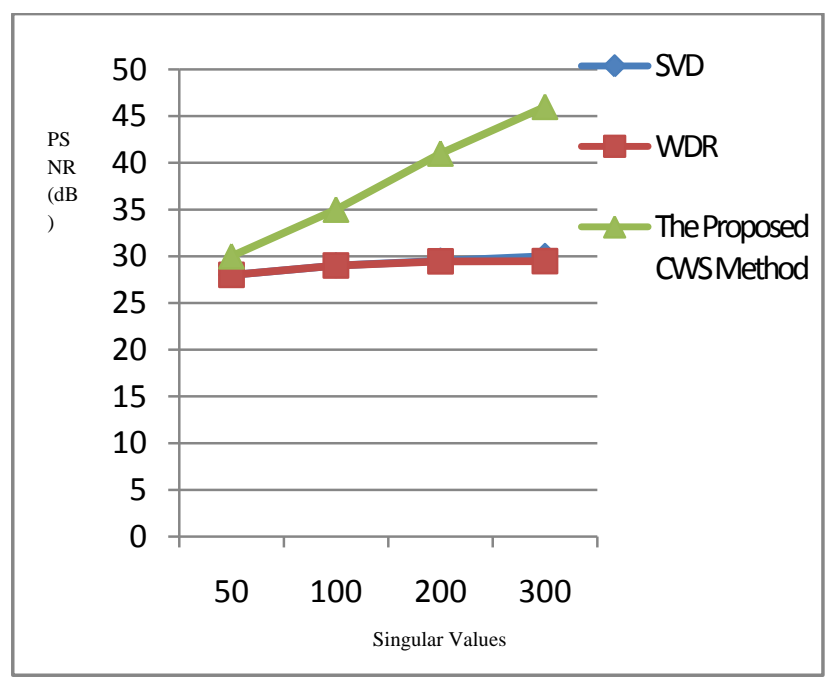

Figure 5: Quality of image for various singular values

The quality of the proposed CWS method for various singular values is shown in Figure 5. The graph explores the superiority of the proposed compression technique over other existing techniques. The quality of the images gets increasing over increase in singular values. The proposed technique is applied on huge images to predict their performance. The average PSNR value is $45.25 \mathrm{~dB}$ for singular value 300 .

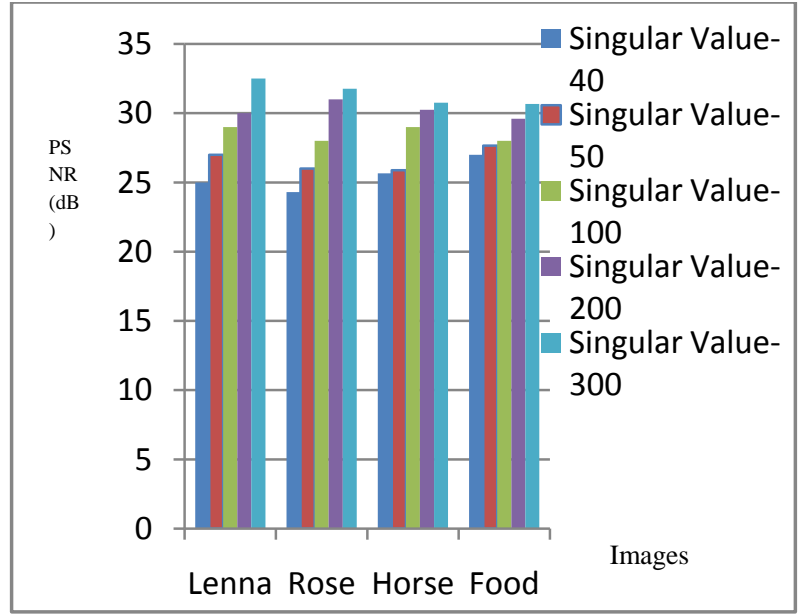

Figure 6: Average performance of the proposed CWS technique for various images

Figure 6 shows the average PSNR value for four images at various singular values. The average PSNR value is $30 \mathrm{~dB}$ for those images. Also it is found that the quality of the images increases with increase in singular values.

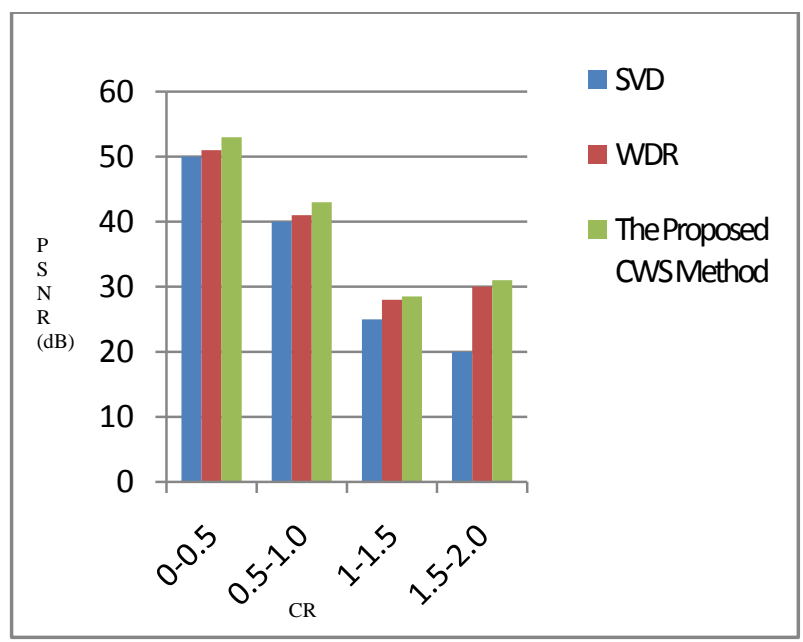

Figure 7: Average performance of the proposed CWS technique at various compression ratios

Figure 7 maps their average performance over various compression ratios. The average PSNR value of the proposed technique reaches 30 when the compression ratio is between $0.5-1$. 


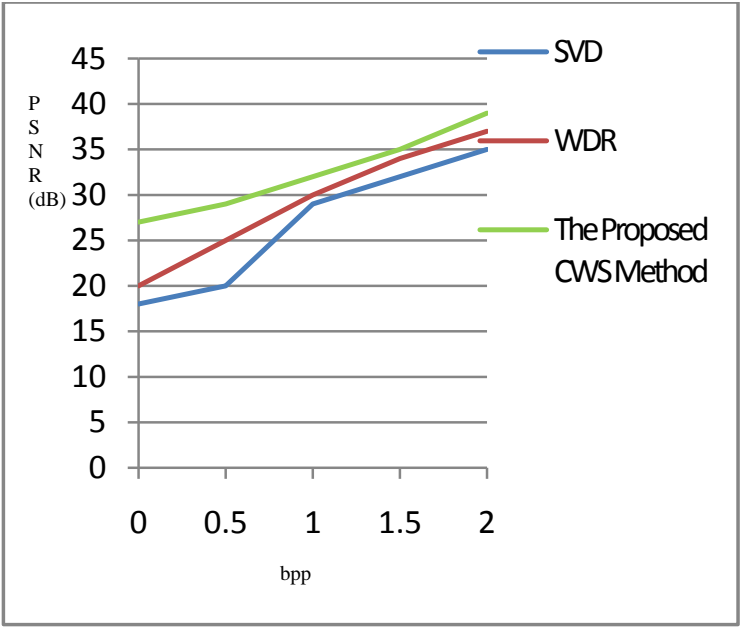

Figure 8: Performance of proposed CWS method over other techniques

The quality of the proposed technique over bits per pixel is depicted in Figure 8. The bits per pixel increases over increase in the PSNR values. Even though the quality is less for lower bits, the proposed method shows better improvement over other existing techniques.

\section{CONCLUSION}

A new CWS image compression technique using WDR and SVD is proposed. First, the image was compressed using WDR, which offers high compression ratios and then the reconstructed image is applied to perform SVD to yield good PSNR. The results were compared with various other lossy techniques in terms of some compression metrics. The proposed technique is tested for 100 images in order to measure the quality. The average PSNR value gets higher when compared with other techniques. Thus it is found that the proposed system shows the superiority over other techniques. Regarding future work directions, aiming at further enchancing the quality of reconstructed image and to extend this research to medical MRI brain images.

\section{REFERENCES}

[1] Moonen M, Van Dooren P, Vandewalle J. Singular value decomposition updating algorithm for subspace tracking," SIAM Journal on Matrix Analysis and Applications, 1992.

[2] Konda T, Nakamura Y. A new algorithm for singular value decomposition and its parallelization. Parallel Comput., 2009,2(1).

[3] Andrews H C, Patterson C L.Singular value decompositions and digital image processing. IEEE Trans.On Acoustics, Speech, and Signal Processing, 1976: 26-53.

[4] Julie Kamm L. SVD-Based Methods For Signal And Image Restoration. PhD Thesis, 1998

[5] Yang J F, Lu C L. Combined Techniques of Singular Value Decomposition and Vector Quantization for Image Coding. IEEE Trans. Image Processing,1995, 4(8) :1141 -11 .

[6] Anzhou Hu, Rong Zhang, Dong Yin, Yibing Zhan. Image quality assessment using a SVD-based structural projection. Signal Processing: Image Communication, 2014: 293-302.

[7] Pratishtha Gupta, Purohit G N, Varsha Banshal. A survey on image compression techniques. International journal of Advanced Research in Computer and Communication and Engineering, 2014,3(8):7762-7768.

[8] Chien C S, Shih Y T and Chuana C Y, An adaptive parameterized block-based singular value decomposition for image de-noising and compression. Applied mathematics and computation.,2012:10370-10385.

[9] Samruddhi Kahu, Reena Rahate. Image compression using Singular Value Decomposition. International Journal of Advancement in Research \& Technology, 2013: 244-248.

[10] Pinto S J, Gawande J P. Performance analysis of medical image compression techniques. IEEE conference publications, 2012:1 - 4 . 